\title{
PROSPECTS OF POSITRONIUM SPIN ROTATION USING FOR MEDIUM INVESTIGATION
}

\author{
V.G. Baryshevsky and O.N. Metelitsa \\ Institute of Nuclear Problems, Belarussian State University \\ 11 Bobruiskaya Str., Minsk, 220050, Belarus
}

\begin{abstract}
The feasible applications of a new positron spectroscopy technique based on measuring of the time oscillation under positronium annihilation is analyzed.
\end{abstract}

PACS numbers: $36.10 . \mathrm{Dr}$

As has been shown in Refs. [1,2] the anisotropy in the angular distribution of the decay photons with respect to the positronium (Ps) spin direction leads to the appearance of the time oscillations under a positronium decay in a magnetic field. These oscillations are caused by the oscillations of positronium spin in an external magnetic field. It allows us to call the experiments on the registration of time oscillations as the PsSR (positronium spin rotation) experiments by analogy with the well known $\mu \mathrm{SR}$ (muon spin rotation) method.

The detailed theory of time oscillations has been developed by us in Ref. [3]. It permits to find the only feasible geometry of experiments which gives the possibility of observation of these oscillations [4]. Recently these experiments were repeated $[5,6]$.

It is necessary to note that the goal of our experiments [4] and of experiments $[5,6]$ was to check existence of oscillations but not to investigate the matter. However, upgrading of time resolution of equipment to 1-2 ns and measurements of oscillations in a uniform $(\approx 0.1 \%$ in target volume) magnetic field allows to use this technique in some unique experiments. Below we consider the basic concepts of PsSR experiments. Also, we proposed feasible application of PsSR technique.

Let us consider a positronium atom in a magnetic field in a nonstationary state described by a spin wave function

$$
|\psi(t)\rangle=\sum_{n=1}^{4} C_{n}|n\rangle \exp \left(-\mathrm{i} E_{n} t-0.5 \gamma_{n}\right)
$$

where $|n\rangle, E_{n}$ and $\gamma_{n}$ are the spin wave function, energy and decay width of $n$-th stationary state of positronium in a magnetic field, respectively, $\hbar=1$. 
In the decay rate measurements we register the delay coincidence of nuclear photons (for example, formed by $\mathrm{Na}^{22}$ positron source) and one (or more) of decay photons. The decay rate registered by "small" detectors is proportional to the squared annihilation amplitude

$$
\begin{aligned}
|M|^{2} & =\left|\sum_{n=1}^{4} C_{n} M_{n} \exp \left(-\mathrm{i} E_{n} t-\frac{1}{2} \gamma_{n}\right)\right|^{2} \\
& =\sum_{m, n}^{4} C_{m} C_{n}^{*} M_{m} M_{n}^{*} \exp \left[-\mathrm{i}\left(E_{m}-E_{n}\right)-\frac{1}{2}\left(\gamma_{m}-\gamma_{n}\right)\right],
\end{aligned}
$$

where $M_{i} \propto\langle 3 \gamma|\widehat{T}| i\rangle$ is the amplitude of three-photon decay of $i$-th stationary state of positronium in a magnetic field. A positronium atom is formed in matter by positron and electron which are described by spin matrix of density but not spin wave functions. Hence, in a real experimental situation positronium is described by a spin density matrix $\rho$, but not wave function. Substituting $C_{m} C_{n} \exp (\ldots) \rightarrow$ $\rho_{m n}$ in Eq. (2) we obtain

$$
|M|^{2}=\sum_{i, k}^{4} \rho_{m n}(t) M_{m} M_{n}^{*} .
$$

If ortho-para conversion is negligible, the positronium spin matrix of density takes the form

$$
\rho_{m n}(l)=\rho_{m n}(0) \exp \left[-\mathrm{i}\left(E_{m}-E_{n}\right)-\frac{1}{2}\left(\gamma_{m}-\gamma_{n}\right)\right],
$$

where $\rho_{m n}(0)$ is the spin density matrix of positronium at the moment of its formation $t=0, \rho_{m n}(0)$ equals the direct product of spin density matrix of positrons entering the target and that of the electrons of matter.

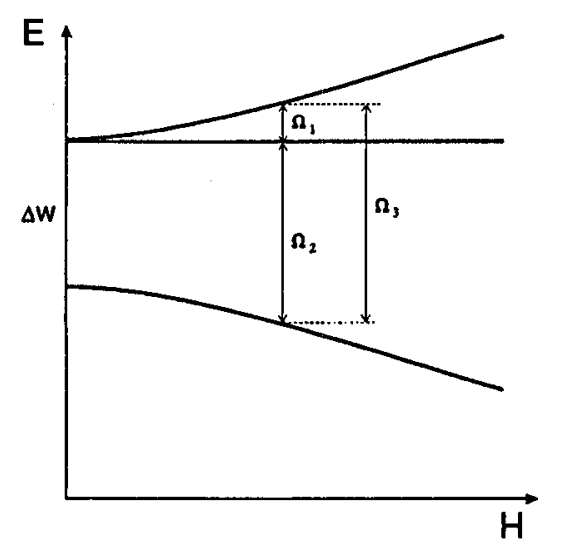

Fig. 1. Oscillation frequencies and the splitting of energies of the positronium levels in a magnetic field. 
It is easy to see from Eqs. (2)-(4) that the squared annihilation amplitude undergoes oscillation at three frequencies, corresponding to splitting energies of positronium levels in the magnetic field (see Fig. 1):

$$
\begin{aligned}
& \Omega_{1}=\frac{1}{2} \Delta W\left[\left(1+x^{2}\right)^{1 / 2}-1\right] \\
& \Omega_{2}=\frac{1}{2} \Delta W\left[\left(1+x^{2}\right)^{1 / 2}+1\right], \\
& \Omega_{3}=\Delta W\left(1+x^{2}\right)^{1 / 2},
\end{aligned}
$$

where $\Delta W$ is the energy of hyperfine splitting of positronium ground state, $x=$ $4 \mu B / \Delta W=2.75 \times 10^{-3} B[\mathrm{~T}], \mu$ is the Bohr magneton, $\Delta W$ is the energy of the hyperfine splitting in a positronium atom. It is much easier to observe the oscillations in a weak $x \ll 1$ magnetic fields at the frequency $\Omega_{1}$, since $\Omega_{2}, \Omega_{3} \approx$ $10^{12} \mathrm{~s}^{-1}$ and terms containing $\Omega_{2}$ and $\Omega_{3}$ reduce to zero after averaging over the detector time resolution $\left(\approx 10^{-10} \mathrm{~s}\right)$. In addition the amplitudes of the oscillations at the frequencies $\Omega_{2}, \Omega_{3}$ in a weak magnetic field are small.

The expressions describing the oscillations under various conditions as well as the best geometry of experiments were considered in our previous papers [3, 4]. We present the expression for coincidence rate $\sigma$ for the case in which only one from the decay photons is registered

$$
\begin{aligned}
\sigma(t) & \propto \Delta \Omega\left\{F_{z z} \exp \left[-\left(\gamma_{q t}+\gamma_{\mathrm{p}}\right) t\right]+\left(F_{x x}+F_{y y}\right) \exp \left[-\left(\gamma_{t}+\gamma_{\mathrm{p}}\right) t\right]\right. \\
& \left.+2 P \sin \theta F_{z y} \sin \left(\Omega_{1} t\right) \exp \left[-\gamma_{\mathrm{p}} t-\frac{1}{2}\left(\gamma_{q t}+\gamma_{t}\right) t\right]\right\},
\end{aligned}
$$

where the solid angle $\Delta \Omega$ subtended by the detector registered decay quanta is assumed to be small, $\theta$ is the angle between the external magnetic field direction (the $O Z$ axis) and the positron average polarization vector. It is assumed that the latter lies in the plane $X Z, P$ is the degree of polarization of the positron entering the target, $\gamma_{g t}=(1 / 4) x^{2} \gamma_{s}+\gamma_{t}$ is the decay rate of quasitriplet positronium, $\gamma_{t}$, $\gamma_{s}$ and $\gamma_{p}$ are the decay rates of ortho- and parapositronium atom and the pick-off annihilation rate, respectively,

$$
F_{i k}=A \delta_{i k}+D n_{i} n_{k},
$$

$\delta_{i k}$ is the Kronecker $\delta$-symbol, $n$ specifies the direction toward the detector. The form of symmetrical Cartesian tensor $F$, i.e. the magnitudes of coefficients $A$ and $B$ depend on a sort of detector. For the case of real experimental geometry we obtain these magnitudes by computer simulation. If the detector efficiency is the same for all photons with energies from 0 to $511 \mathrm{keV}$, we can present the analytical result [3]:

$$
A=2\left(\pi^{2}-9\right)-\frac{1}{3}, \quad D=-2\left(\pi^{2}-9\right)+1 .
$$

The terms $\sim x$ in Eq. (6) are dropped.

Figure 2 gives the cross-section (6) as a function of detector position and time. 


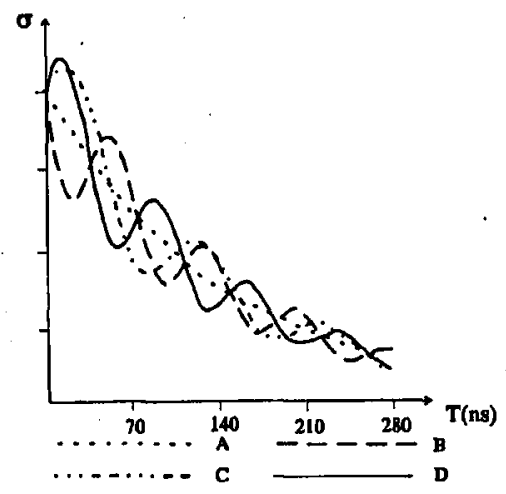

Fig. 2. Time dependence of the positronium three-photon annihilation cross-section (arbitrary units) in a magnetic field at various detector orientations: $A-\alpha=0$, $B=0.059 \mathrm{~T} ; B-\alpha=\pi / 2, \beta=\pi / 4, B=0.059 \mathrm{~T} ; C-\alpha=\pi / 2, \beta=\pi / 4$, $B=0.05 \mathrm{~T} ; D-\alpha=\pi / 2, \beta=-\pi / 4, B=0.059 \mathrm{~T}$.

As follows from Eqs. (6)-(8), the oscillation modulation depth $h$ being equal to the ratio of the oscillation amplitude to the value of nonoscillating term at $x^{2} \gamma_{s} \ll \gamma_{t}$ is defined by the following expression:

$$
h=\frac{2\left(\pi^{2}-9\right)-1}{2\left(\pi^{2}-9\right)} P|\sin \theta \sin 2 \beta \sin \alpha| \approx 0.213 P|\sin \theta \sin 2 \beta \sin \alpha|,
$$

where $\beta$ and $\alpha$ are the polar and azimuthal angles specifying the direction towards the detector. The oscillation amplitude reaches its maximum when the positrons entering the target are polarized perpendicular to the external magnetic field direction $(\theta=\pi / 2)$, and the counter registering decay quanta is placed at the angle $\beta=\pi / 4$ to the magnetic field direction in the plane normal to the positron polarization vector $(\alpha=\pi / 2)$. Oscillation phases, observed at angles $\beta=\pi / 4$ and $\beta=3 \pi / 4$ ( $\alpha=$ const) differ by $\pi$. This feature had been used for observation of oscillations [4-6].

In the PsSR experiments as well as in $\mu \mathrm{SR}$ experiments we can measure the oscillation frequency and amplitude, oscillation dumping. This leads to the possibility of the following application of PsSR method:

- measurements of $n=1$ hyperfine structure of positronium

As we can see from Eq. (1) the oscillation frequency is proportional to the energy of hyperfine splitting of positronium ground state. Thus, the measurements of this frequency allow to find the magnitude of this splitting in various media. The measurements of hyperfine structure of positronium in vacuum may be also of interest in view of QED testing [7].

- measurements of ortho-para conversion rate in gases and aerogels As can be shown, the ortho-para conversion leads to the oscillation dumping with the rate $3 \nu$, where $\nu$ is the ortho-para conversion rate. Thus, the 
measurement of the oscillation dumping is a direct way (in contrast to [8]) to measure the conversion rate.

- measurements of exchange splitting of $m= \pm 1$ levels of positronium in optically polarized media

It was shown [2] that orthopositronium $m= \pm 1$ levels are splitted (see Fig. 3) in a polarized media due to exchange interaction between the electron of positronium and electrons of polarized media. Unfortunately, the direct observation of $\Omega_{0}$ frequency is impossible [9] since it is necessary that the polarization of media electrons is normal to the magnetic field direction. However, changing detector placement with respect to positron polarization we can observe oscillations at the frequencies $\Omega_{1}, \Omega_{-1}$ as well as the beating at the difference of these frequencies [9].

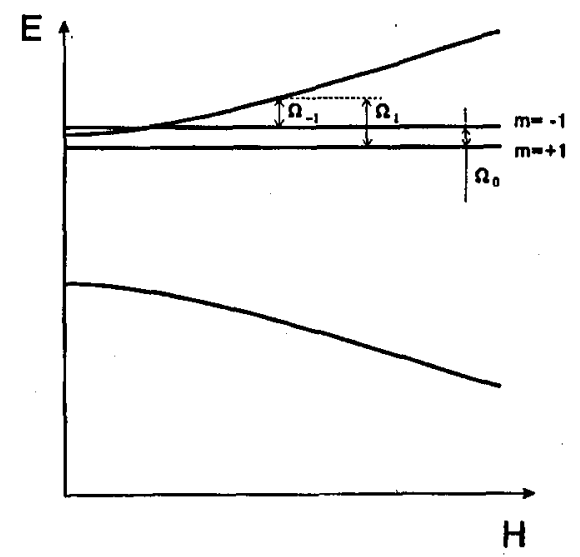

Fig. 3. The splitting of the positronium levels in a polarized medium in a magnetic field.

\section{Acknowledgment}

The research described in this publication was made possible in part by Grant No. MWU000 from the International Science Foundation and by Belarussian Foundation of Fundamental Research.

\section{References}

[1] V.G. Baryshevsky, Dokl. Akad. Nauk BSSR 16, 445 (1966).

[2] V.G. Baryshevsky, Phys. Status Solidi B 124, 619 (1984).

[3] V.G. Baryshevsky, O.N. Metelitsa, V.V. Tichomirov, J. Phys. B, At. Mol. Phys. 22, 2835 (1989).

[4] V.G. Baryshevsky, O.N. Metelitsa, V.V. Tichomirov, S.K. Andrukhovich, A.V. Berestow, B.A. Martsinkevich, E.A. Rudak, Phys. Lett. A 136, 428 (1989). 
[5] V.V. Baranov, A.V. Berestov, B.A. Martsinkevich, E.A. Rudak, A.S. Talat, Izv. Rus. Acad. Sci., Ser. Fiz. 58, 137 (1994).

[6] S. Fan, C.D. Beling, S. Fung, in: 10th Int. Conf. on Positron Annihilation, Book of Abstracts, Beijing 1994, p. 216.

[7] A. Rich, Rev. Mod. Phys. 53, 127 (1981).

[8] A. Bisi, G. Consolati, G. Cambarini, L. Zappa, Nuovo Cimento B 65, 442 (1981).

[9] O.N. Metelitsa, Ph.D. Thesis, Belarussian State University, Minsk 1991, p. 210. 